\title{
UM MODELO AFETIVO DE APRENDIZAGEM PARA O ESTUDO DE ANÁLISE COMBINATÓRIA
}

\author{
Márcia Jussara Hepp Rehfeldt \\ Maria Lucia Pozzatti Flôres \\ Magda Bercht
}

Resumo: Este artigo apresenta um sistema para o apoio ao ensino e a aprendizagem de análise combinatória. A área escolhida decorre da verificação que este componente curricular é um dos que mais causa frustração e promove raciocínios inadequados envolvendo o raciocínio lógico, nos diversos cursos de graduação. A escolha também se justifica pela relação direta entre dificuldade de aprendizagem e motivação. Propõem-se auxiliar nas dúvidas dos alunos e motivá-los frente a dificuldades a fim de minimizar o índice de desistências e aumentar o grau de satisfação. Um modelo afetivo de cada aluno é atualizado durante cada interação, utilizando-se a motivação do estudante em relação às tarefas em desenvolvimento. A validação do sistema é realizada com alunos dos cursos de Administração nas instituições de ensino superior onde as autoras lecionam.

Palavras-chaves: modelo afetivo, motivação, sistema de apoio ao ensino e aprendizagem, ensino de análise combinatória.

Abstract: This article presents a system used to support teaching and learning of Combination Analysis. The scope of studying was choosed because this curriculum component is one of the most frustrate for students and is the responsible by inadequate development of the logical reasoning, in the various courses of graduation. The selection also is justified by the direct relation between learning difficulty and motivation. The system is proposed to help students in their doubts and motivate in face of some difficulties, thus minimizing the index of desistance and increase the satisfaction level. An affective model for every student is refreshed during every iteration, utilized the student's motivation in relation with the task developed. The system's validation is done with students that courses Administration in the Institutions where the authors teach.

Keywords: affective model , motivation, system to support the teaching and learning, teaching to Combination Analysis.

\section{1-INTRODUÇÃO}

Cada vez mais tem se verificado que os estudantes que ingressam em cursos do ensino superior têm problemas nas competências mínimas em seu aprendizado. Aliada a esta realidade percebe-se a falta de conhecimento dos processos cognitivos dos alunos, por parte dos educadores. Um exemplo disso ocorre quando o professor apresenta seu material didático de uma maneira linear, sem levar em consideração os conhecimentos que os alunos trazem e têm. Assim, nem sempre o aluno entende o que o professor fala. A falta de compreensão do significado traz como consequiência àquilo que alguns autores definem como erro. Para Brosseau (1982 apud Freitas, 2002), "erros não são simples ausências de conhecimentos: expressam conhecimentos mal formados que depois se tornam resistentes". Segundo Brosseau (1982 apud Silva, 1997), "os erros 
em um mesmo sujeito estão ligados entre si por uma causa comum: uma maneira de conhecer, uma concepção característica, um conhecimento antigo e que tem êxito em todo um domínio de ações".

a) Estudos de Gómez-Granell (1998) descritos por Souza (2005), apontam que uma boa parcela dos erros cometidos pelos alunos deve-se ao fato de ter sido baseado muito mais na aplicação de regras que na compreensão do significado. Os alunos aprendem a manipular símbolos sem se aperceberem do sentido que eles têm, aplicam as regras que lhes foram ensinadas, mas não são capazes de conectá-las nem com seu conhecimento procedimental nem com o conceitual.

b) Estes alunos, aplicadores de regras e, muitas vezes pouco motivados, com um tempo mínimo para estudar são os que encontramos em maior número nas instituições superiores do país. No entanto, precisam ser estimulados para superar suas dificuldades, pois do contrário aumentam a lista de desistentes.

c) Neste sentido, o projeto aqui proposto, embasado em Kort, Reilly e Picard para fundamentar o Modelo Afetivo da Inter-relação entre Emoções e Aprendizagem, e em Joseph E. Beck para detectar se um estudante está desmotivado em relação a resolução de um problema poderá trazer resultados que venham a reduzir o número de desistências e motivar os alunos na busca de soluções para suas dificuldades, tornando o aluno mais autônomo e co-responsável pela sua formação.

Este artigo apresenta a seguir um item que discute os problemas que levam as autoras a pesquisar e desenvolver tal proposta. $O$ terceiro item destacará a fundamentação, enquanto o quarto item apresenta a descrição do sistema com suas ferramentas e funcionalidades. O quinto item apresenta a forma de validação do sistema e das experiências para coleta dos dados para análise e verificação das hipóteses.

\section{2- O PROBLEMA}

Atualmente, os processos seletivos das instituições privadas de ensino superior não estão mais selecionando candidatos e sim apenas classificando-os. Como são instituições nas quais o ensino não é gratuito, implica em pagamentos de mensalidades que são oriundas, na sua grande maioria, dos próprios estudantes.

Essa realidade implica em um número reduzido de horas que os alunos efetivamente dedicam ao estudo, o que gera baixo aproveitamento. Além do mais, os horários das monitorias e aulas de reforço, nem sempre estão adequados à disponibilidade de horário dos estudantes. Sendo assim, o aluno de graduação, na sua grande maioria, dedica-se aos estudos nos finais de semana, nos horários do meio-dia ou após as aulas. E quando se depara com dificuldades, não tem a quem recorrer. Como consequiência, o aluno desiste ou reprova, acarretando um prejuízo para si e para a Instituição.

Diante desta realidade torna-se necessária e urgente um sistema que possa além de auxiliar nas dúvidas dos alunos, motivá-los a enfrentar as dificuldades e aumentar o grau de satisfação dos alunos. Inicialmente, o projeto que está sendo proposto será aplicado para o estudo da análise combinatória, visto que este componente curricular é um dos que mais causa frustração e promove raciocínios inadequados na área que envolve raciocínio lógico nos diversos cursos de graduação.

A escolha deste assunto justifica-se pela relação direta entre dificuldade de aprendizagem e motivação, ou seja, aluno que não está motivado para resolver problemas de análise combinatória, normalmente apresenta dificuldades de aprendizagem e, por outro lado, a dificuldade de aprendizagem implica em desmotivação. Além disso, resolver problemas em análise combinatória não é uma tarefa muito simples, pois facilmente o aluno confunde arranjo e combinações, dois 
importantes conceitos, acarretando erros significativos. O tema gera muitas dúvidas e nem sempre as questões estão absolutamente claras, o que causa muita confusão no raciocínio do aluno. E pela interpretação errônea, constrói um raciocínio inadequado para resolver o problema. Devido a esta situação ele se desmotiva, não refaz mais o raciocínio e, conseqüentemente, não aprende o conteúdo, falhando no seu processo de aprendizagem.

Neste projeto as autoras propõem responder a seguinte questão: alunos que interagem com um sistema motivador e problematizador sentem-se mais motivados frente a repetidas falhas na resolução de problemas de análise combinatória?

\section{3-EMOÇÕES NO PROCESSO DE APRENDIZAGEM:}

Kort, Reilly e Picard (2001) discutem o papel das emoções no processo de aprendizagem e procuram tirar conclusões sobre possíveis modelos que integram os aspectos cognitivos e os afetivos. Esses autores têm como enfoque o ensino de áreas científico-tecnológica, como a matemática e as engenharias. De uma maneira geral, quando os professores apresentam o seu material didático, o fazem de uma maneira polida e linear, omitindo o processo natural de aprendizagem. Em particular, são omitidos os aspectos relacionados com os erros, com a confusão e com a frustração inerente a algumas fases do processo.

A teoria de desenvolvimento cognitivo proposta por Piaget (1973), ajuda a compreender que o pensamento matemático não é, em essência, diferente do pensamento humano mais geral, no sentido de que ambos requerem habilidades como intuição, senso comum, apreciação de regularidades, senso estético, representação, abstração e generalização, etc... A diferença que pode ser considerada é no universo de trabalho: na Matemática os objetos são de caráter abstrato e são rigorosos os critérios para o estabelecimento de verdades. A teoria de Piaget procura explicar o complexo processo através do qual se dá o desenvolvimento das funções cognitivas da inteligência. Esse processo se dá em diversos estágios, onde Piaget (1981) mostra a contínua evolução das estruturas mentais, e cujo estado mais avançado se caracteriza pelo pensamento formal abstrato.

Os desequilíbrios entre experiência e estruturas mentais é que fazem o sujeito avançar no seu desenvolvimento cognitivo e conhecimento, e Piaget (1981) procura mostrar o quanto este processo é natural. O novo objeto de conhecimento é assimilado pelo sujeito através das estruturas já constituídas, sendo o objeto percebido de uma certa maneira; o 'novo' produz conflitos internos, que são superados pela acomodação das estruturas cognitivas, e o objeto passa a ser percebido de outra forma. Neste processo dialético é construído o conhecimento.

Alguns professores são extremamente aptos em reconhecer o estado emocional dos seus alunos e, baseados na sua observação, agem de forma a influenciar positivamente o seu processo de aprendizagem. Mas, qual é a melhor forma de atuar em cada situação? Como pode um aluno que atravessa uma situação de insucesso na sua aprendizagem, voltar a uma situação produtiva e gratificante?

Kort, Reilly e Picard (2001) ao verificarem que os professores podem interpretar os sinais emotivos com diferentes níveis de precisão começaram a interessar-se pela possibilidade de computadores virem a ser capazes de identificar expressões afetivas. Estes sinais emotivos, capturados através de agentes inteligentes, podem ser uma boa opção para a inclusão do fator emoção na aprendizagem. Esses autores propõem um modelo que descreve uma gama de estados emocionais que ocorrem durante a aprendizagem.

O modelo de Kort et alli - Modelo Afetivo da Inter-relação entre Emoções e 
Aprendizagem- identificou cinco eixos básicos os quais correspondem a estados emocionais específicos que se situam entre dois extremos de um espectro. Estas seis dimensões, a que chamam de "Eixos", assim como os pólos de cada espectro são: Ansiedade - Confiança, Aborrecimento - Fascínio, Frustração - Euforia, Desanimado Encorajado, Terror - Encantamento, Humilhação - Orgulho.

Este modelo é único em termos do enquadramento e do conjunto de emoções que é tratado. Contudo, os autores afirmam que esta abordagem não é definitiva e que terá ainda que ser avaliado cada Eixo e o conjunto de emoções que lhe está associado. Este conjunto depende da cultura e do estado de desenvolvimento de cada aprendiz.

O mundo das emoções é altamente complexo e pode conter outras misturas de palavras e de conceitos além das aqui referidas. $\mathrm{O}$ desafio é trabalhar com um pequeno número de variáveis e ver qual a sua aderência à realidade.

A visualização do modelo afetivo:

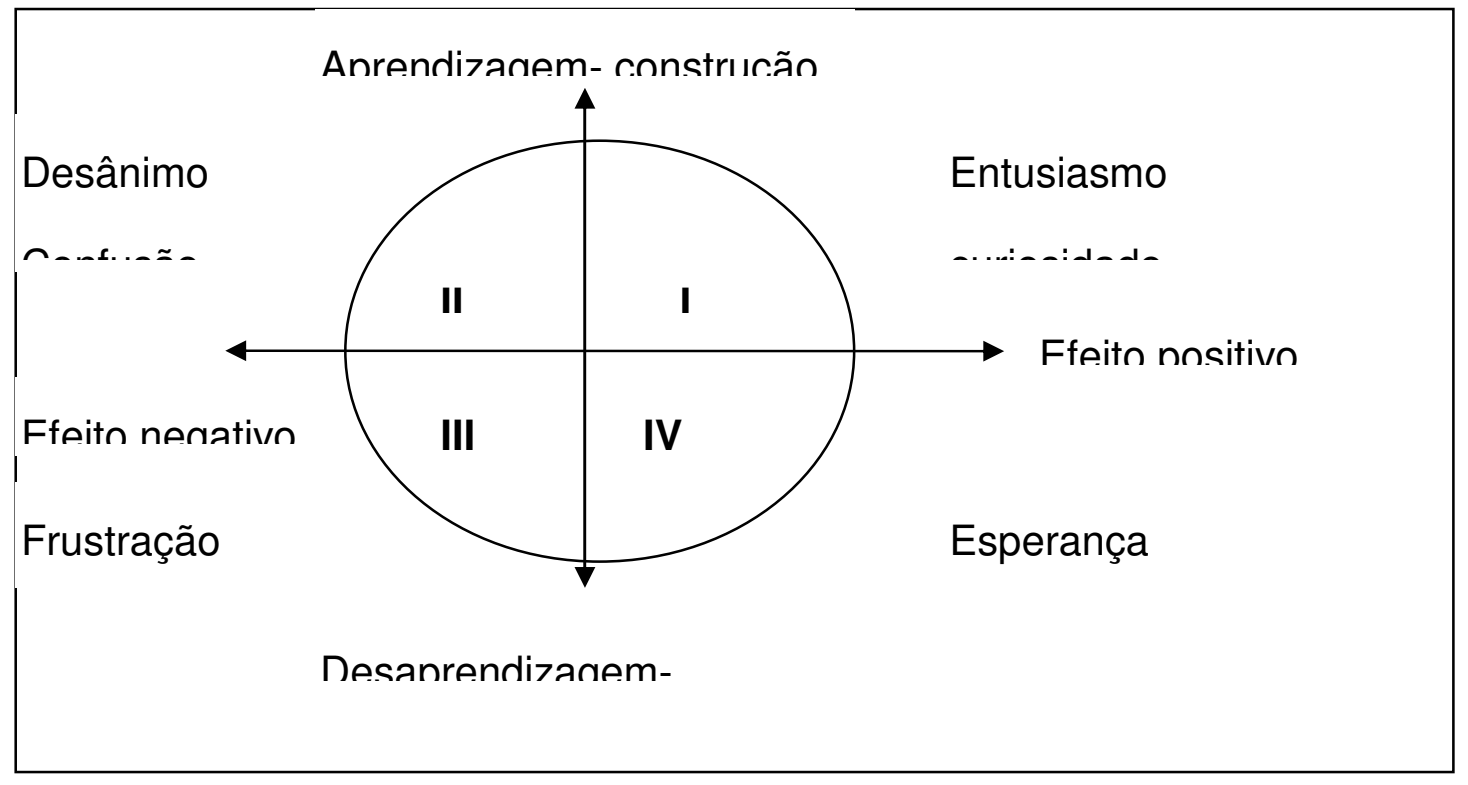

O eixo da Aprendizagem, o eixo vertical, corresponde ao processo de construção/desconstrução de conceitos. O eixo Emocional, o horizontal, corresponde a uma das seis dimensões das emoções básicas consideradas neste modelo. Para a direita, temos os aspectos agradáveis, positivos, de prazer, da emoção e, para a esquerda, fica o pólo negativo que corresponde a sensações mais desagradáveis, de desprazer. Adicionalmente, este modelo incorpora um terceiro eixo, o Eixo do Conhecimento, vertical em relação ao plano. É em redor deste eixo que se realiza o movimento entre os quatro quadrantes, o qual efetua uma órbita em espiral em torno do Eixo do Conhecimento.

No quadrante I, a antecipação e a expectativa são grandes, enquanto o estudante constrói as suas idéias e conceitos e os experimenta. No quadrante II, a taxa de conhecimento diminui e as emoções negativas emergem à medida que o progresso não é tão rápido como esperado. No quadrante III, o estudante tenta eliminar as idéias e conceitos que não se adequavam ao problema. No quadrante IV o estudante recupera a esperança, o entusiasmo e adota uma atitude positiva à medida que foram removidos os conceitos improdutivos e ineficazes, reiniciando-se o ciclo. Salienta-se que a órbita não se fecha sobre si mesma, mas move-se para cima ao longo da terceira dimensão, o eixo do conhecimento. 
A intenção dos autores deste modelo não é de que o aluno esteja sempre no quadrante I, mas sim que o ajude entender o caráter e a natureza cíclica da aprendizagem.

No artigo Using response times to model student disengagement, Joseph E. Beck (2004) propõe um modelo para detectar se um estudante está desmotivado em relação a resolução de um problema. O modelo proposto é baseado na teoria de resposta por item (IRT) e usa como entrada: a dificuldade da questão, quanto tempo o estudante leva para responder e simultaneamente se a resposta está correta. A partir destes dados, o modelo determina a probabilidade de um estudante estar ativamente engajado em tentar responder a questão.

Beck fez três suposições nesse modelo: quais dados incluir para construir um modelo; a fórmula matemática do modelo; e como os estudantes gerariam as respostas.

Ainda, foram consideradas quatro regiões gerais:

-região A, onde o estudante não tem ganho nenhum (chuta);

-região $\mathrm{B}$, a performance do estudante é aumentada à medida que gasta mais tempo;

-região C, a performance atinge um platô;

-região $\mathrm{D}$, a performance diminui gradualmente conforme gasta mais tempo antes de responder a questão.

Pelas regiões $\mathrm{A}, \mathrm{B}$ e $\mathrm{C}$, a performance com respeito ao tempo é similar com uma curva logística, e usa-se a teoria das respostas dos itens (IRT) como um ponto de partida dessa modelagem. A teoria de resposta de itens (IRT) fornece uma estrutura para predizer a probabilidade de um estudante com uma proficiência particular responder uma questão corretamente. Para fazer este cálculo, assume-se que os estudantes têm dois métodos de gerar respostas:

-se o estudante é desmotivado, então ele trabalha com uma probabilidade de $25 \%$ de estar correto (desde que existe quatro opções de respostas);

-se o estudante é motivado, ele responde a pergunta com uma probabilidade de existência igual à correta na região $\mathrm{C}$.

Dada essas suposições, a probabilidade do estudante ser desmotivado é $p=\frac{\text { Faixa sup erior }- \text { Performanceesperada }}{\text { Faixa sup erior }- \text { Faixa inf erior }}$

Para estimar a probabilidade da resposta de um estudante estar correta, foram usados três tipos de modelo. $\mathrm{O}$ primeiro modelo IRT é dado por: $p($ corret $\mid \theta)=c+\frac{1-c}{1+e^{-a(\theta-b)}}$

Sendo que na equação: $\theta$ é a proficiência do estudante; a é o parâmetro de discriminação e determina a normalidade da inclinação da curva logística; b é o parâmetro de dificuldade e controla quanto para a direita ou para a esquerda a curva está inclinada; c é o parâmetro aleatório e fornece uma faixa inferior à curva. Quando os itens são questões de múltipla-escolha e com quatro respostas, atribuímos $\mathrm{c}=0,25$ (ou $25 \%)$.

No segundo modelo foi modificada a fórmula padronizada em vários caminhos. Em vez de tomar a proficiência do estudante como entrada, foi usado tempo de resposta. Também, foram estimados parâmetros de item para cada pergunta, como um modelo do IRT puro poderia fazer, desde o número de vezes de tempo que uma pergunta foi vista. Portanto, foi estimado discriminação e parâmetro de dificuldade de item para cada tipo de questões. Desde que o parâmetro de dificuldade não pode capturar as diferenças entre perguntas, também foi incluído o comprimento da questão e escolha de resposta (como número de caracteres). Perguntas longas são mais difíceis que questões mais curtas. A 
forma do modelo modificado é: $p\left(\right.$ corret $\left.\mid r t, L_{1}, L_{2}\right)=c+\frac{d-c}{1+e^{-a\left(-r t+b\left(L_{1}+L_{2}\right)\right.}}$

Nesta equação, os parâmetros a, b e c têm o mesmo significado do modelo IRT. $\mathrm{O}$ parâmetro d representa a faixa superior em performance, e $\mathrm{L}_{1}$ e $\mathrm{L}_{2}$ são o número de caracteres na pergunta e em todas as questões de respostas, respectivamente. O d é um parâmetro igual a performance máxima. Estimamos os parâmetros a (discriminação) e b (dificuldade) separadamente para cada tipo de questão, usando a função não linear de regressão SPSS.

O terceiro modelo considera a variabilidade entre estudantes, e para estimar os parâmetros a, b e d para cada estudante e cada tipo de pergunta. Estudantes viram uma média de 33.5 e uma mediana de 22 questões em que eles responderam em menos de 7(sete) segundos. Portanto, primeiro foi estimado os parâmetros para cada tipo de pergunta, e então dois parâmetros adicionais para cada estudante que se aplicam através de todos os tipos de questões. $\mathrm{O}$ novo modelo é dado por: $p\left(\right.$ corret $\left.\mid r t, L_{1}, L_{2}\right)=c+\frac{\text { precisão }(1-d)+d-c}{1+e^{-a\left(-r t+\text { velocidadé } b\left(L_{4}+L_{2}\right)\right.}}$

onde a precisão e a velocidade são os parâmetros específicos para estudarem. $\mathrm{O} 1^{\circ}$ parâmetro adicional, velocidade, mede as diferenças em velocidade de leitura do estudante para ajustar o impacto do comprimento da pergunta e resposta escolhida. $\mathrm{O} 2^{\circ}$ parâmetro, precisão, é o nível de conhecimento do estudante. Estudantes que sabem mais palavras, ou que estão melhores na eliminação das respostas erradas das opções de resposta.

Para validar o modelo, foram analisados 231 interações de estudantes com a versão 2002/2003 do tutor de leitura. Mostrou-se que a desmotivação é modelada melhor através da estimativa simultânea da proficiência e desmotivação do estudante do que só pela desmotivação. O melhor modelo de desmotivação tem uma correlação de $-0,25$ com os ganhos de aprendizagem do estudante. $\mathrm{O}$ aspecto novo deste trabalho é que ele requer somente dados coletados normalmente por um tutor computacional e o modelo afetivo é validado contra a performance do estudante em uma medida externa.

\section{4-O AMBIENTE PROPOSTO:}

Este projeto visa à construção de um sistema de ensino, cujo domínio de conhecimento é de uma parte da matemática chamada Análise Combinatória, mais especificamente a resolução de problemas de análise combinatória. Este sistema pode ser usado pelo aluno como reforço dentro ou fora da sala de aula.

O comportamento previsto para o aluno é mostrado na figura a seguir: 


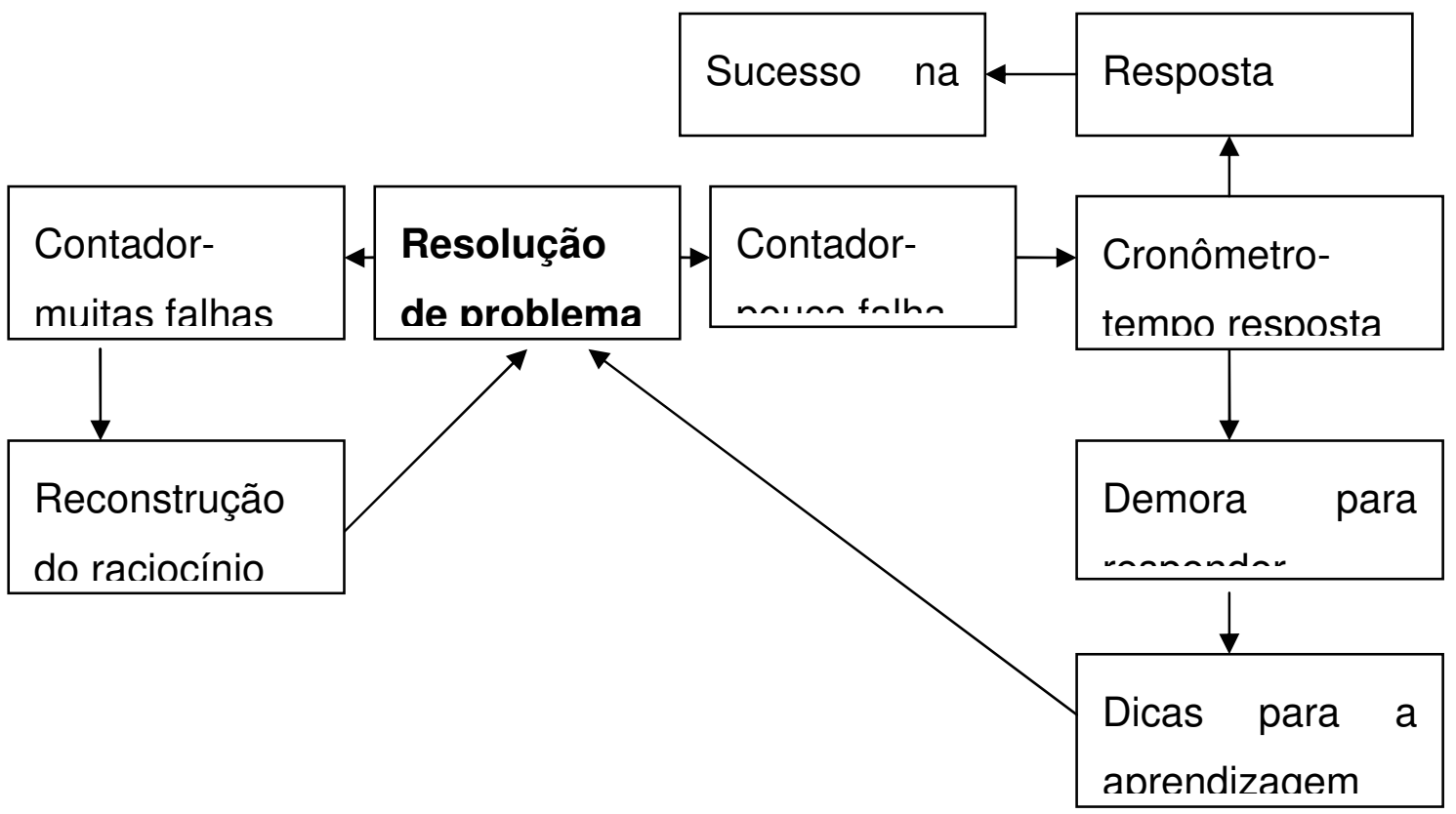

O esquema explica como está sendo pensada uma situação de aprendizagem e qual a interferência do sistema. Um aluno ao ser desafiado usa seus processos cognitivos para resolver um determinado problema, testa-os e verifica o resultado. Podem ocorrer dois fatos: obter sucesso e, conseqüentemente, aprendizado ou seu processo de construção está incorreto e ele comete uma falha. Neste caso, ele necessita reconstruir seu raciocínio e é estimulado para isto, recebendo instruções claras sobre cada passo da resolução do problema, ou ainda, reforçando alguma parte da matéria. Vê-se novamente frente a um problema para resolver, no qual precisa testar novamente sua lógica. Essa metodologia torna-se cíclica enquanto o aluno não obtiver sucesso. As ações por parte do sistema de ensino são:

a)Propor a resolução de um problema a um aluno;

b) Contar o número de vezes que o aluno tentou resolver o problema através de um contador;

c) Se tiver pouca ou nenhuma falha, cronometrar o tempo de resposta;

d) Se a resposta for rápida e certa, a aprendizagem foi bem sucedida;

e) Quando o aluno demora além do tempo determinado pela metodologia de Beck, interferir na resolução do problema, dando dicas sobre como resolvê-lo;

f) Se o contador acusar muitas falhas, reconstruir o raciocínio, mostrar o grau de dificuldade em cada problema e por onde iniciar o pensamento para resolver o problema de contagem;

g) O sistema terá que motivar o aluno a recomeçar o estudo, tantas vezes quantas forem necessárias, até que ele atinja o grau razoável de acertos na resolução de problemas de análise combinatória.

\section{1- ESPECIFICIDADES DA FERRAMENTA}

a)Introdução e Motivação: deve descrever o objetivo para trabalhar com Análise Combinatória, ou com os processos de contagem indireta de todas as possibilidades de ocorrência para determinados acontecimentos. Estes acontecimentos serão os diferentes agrupamentos que podemos formar, a partir dos elementos de um conjunto dado, usando ou não todos os elementos, ditos disponíveis. Há agrupamentos que serão vistos como subconjuntos do conjunto inicial, não interessando a ordem de colocação de seus 
elementos, e há agrupamentos que serão estudados com sucessões, ou seqüências, cabendo distinguir quais os seus elementos e em que ordem estão colocados ou dispostos. Deve-se ter o cuidado para que os objetivos e conteúdos sejam relevantes para o aluno, o que aumenta a chance de se estabelecerem vínculos afetivos entre o sujeito e os objetos.

b)Histórico: mostrar como iniciou o estudo da Análise Combinatória. Mostrar o que é contagem; a diferença entre arranjo, permutação e combinação. Mostrar que a análise combinatória faz parte do nosso dia-a-dia, tais como combinações possíveis de letras e algarismos para formar as placas dos carros, números telefônicos, preenchimento de cartões de loteria, etc, bem como, problemas tecnicamente mais sofisticados, quando se faz uma composição destes recursos da contagem com o cálculo de probabilidade e mesmo com a Estatística.

c)Técnicas de aprendizado: usar técnicas de aprendizado dos tipos: aquisição direta de novo conhecimento (formar uma biblioteca com todo o assunto de Análise Combinatória); aprendizado por descoberta (associação entre um conceito de análise combinatória e sua fórmula); aprendizado baseado em explicações.

\section{d)Arquitetura do Componente de Aprendizado}

.Aprendizagem significativa: construir um sistema para verificar o que o aluno já sabe sobre problemas de contagem, elaborando testes diagnósticos com diferentes níveis de dificuldade sobre problemas de análise combinatória e verificando até que nível de dificuldade o aluno tem condições de resolver.

.Elemento de aprendizado: montar uma biblioteca de conteúdo e exercícios de análise combinatória, numa ordem lógica dos conceitos. Montar uma biblioteca de situações onde aparece algum conceito de análise combinatória e a sua fórmula. Montar uma biblioteca somente de exercícios apropriados a cada conceito, mostrando o desenvolvimento dos mesmos, o nível de dificuldade e a área onde eles são aplicados. Montar uma biblioteca com pastas de testes sobre cada conceito e outra contendo a solução dos teste e como resolvê-los passo a passo.

.Avaliação de desempenho e seleção de ações externas: construir um sistema cuja função é monitorar as ações do estudante (incluindo as afetivas) e atuar dinamicamente, com instruções claras e intervenções adequadas, durante a tentativa do aluno para resolver um exercício ou para auxiliá-lo na resolução de um problema.

.Gerador de problemas: construir um sistema que problematize indicando vários tipos diferentes de problemas a serem resolvidos.

.Crítica: construir um sistema cuja função é direcionar os estudos do aluno dizendo se o aluno está certo ou errado; fazendo sugestões, fazendo elogios, fazendo que o aluno seja motivado a continuar seus estudos ou a voltar e reforçar o que ficou falho. Este agente deverá entrar em ação depois que o aluno tentou resolver por três ou mais vezes o mesmo problema ou tenha demorado mais de 5 minutos para responder a questão pela primeira vez.

.Feedback: para reforçar o aprendizado, construir uma interface para a visualização de exercícios resolvidos, onde o estudante comanda, passo-a-passo, a execução da solução do exercício que aparece na forma de animação, ao mesmo tempo em que uma janela de texto apresenta uma explicação sobre cada etapa da solução.

\section{5- A APLICAÇÃO}

Para verificar a quantidade de vezes que o aluno tentou resolver a questão, será implementado um contador. Toda vez que o aluno tentar por três vezes ou mais acionar a mesma questão e não obtiver sucesso, o sistema deverá auxiliar o aluno, reforçando o aprendizado, dando dicas de como resolver o problema. Se o aluno demorar mais de 5 
minutos para responder a questão, o sistema deverá entrar em ação, fazendo sugestões, fazendo elogios, fazendo com que o aluno seja motivado a continuar seus estudos.

Para validar se o tempo de 5 minutos para dar a resposta é o melhor tempo proposto por Beck e verificar se tentar por três vezes é o número ideal para o contador, será realizado um teste piloto com 60 alunos.

Essa metodologia será aplicada nos alunos dos Cursos Administração nas instituições de ensino superior onde as autoras lecionam, durante um determinado período. Após será reavaliado todo o ambiente de aprendizagem com vistas à melhoria.

\section{6-CONSIDERAÇÕES FINAIS}

Este projeto, embora não implementado, espera contribuir para reflexões acerca da implementação de sistemas para auxiliar na aprendizagem dos alunos, principalmente nas instituições de ensino nas quais os alunos têm dificuldades em encontrar espaço e tempo para dedicar-se aos estudos.

Os referenciais teóricos sustentam que o tema afetividade é de suma importância para o avanço da aprendizagem dos alunos.

Além disso, a metodologia proposta por Beck para detectar se um estudante está desmotivado em relação a resolução de um problema encontrou poucos reflexos nos meios acadêmicos. O modelo de Beck mostrou que a desmotivação é modelada melhor através da estimativa simultânea da proficiência e desmotivação do estudante.

\section{2- REFERÊNCIAS BIBLIOGRÁFICAS}

BECK, Joseph. Using response times to model student disengagement. Proceedings of the ITS2004 Workshop on Social and Emotional Intelligence in Learning Environments, Pittsburgh, USA, 2004. Disponível em http://www.ri.cmu.edu/pubs/pub_4993.html. acesso em: 12 jul 2005.

FREITAS, Marcos Agostinho de. Equação do $1^{\circ}$ grau: métodos de resolução e análise de erros no ensino médio. Dissertação de mestrado. PUC/SP, 2002.

KORT, Barry e REILLY, Rob. A pedagogical model for teaching scientific knowledge. In: $32^{\text {nd }}$ ASEE/IEEE Frontiers in Education Conference. Período de 6 a 9 de novembro de 2002. Boston, MA. Disponível em: http://fie.engrng.pitt.edu/fie2002/papers/1117.pdf. , acessado em 15 de maio de 2005.

Media Lab do MIT- grupo de "Affective Computing". Disponível em: http://affect.www.medialab.mit.edu , acessado em 15 de maio de 2005.

PIAGET, Jean. (1954) Intelligence and affectivity: their relationship during child development. Annual Reviews, Palo Alto-CA, (ed.USA, 1981).

PIAGET, Jean. Inteligencia y afectividad. Buenos Aires: Aique, 2001.

Piaget, J. Comments in Mathematical Education, em A.G.Howson (ed) Proceedings of the Second International Congress on Mathematical Education, Cambridge University Press, 1973.

SILVA, Maria José Ferreira da. Sobre a introdução do conceito de número fracionário. Dissertação de mestrado. PUC/SP, 1997.

SOUZA, Sueli Spolador Simões de. O papel construtivo do erro no processo de ensino e aprendizagem da matemática. Disponível em:<www.sbempaulista.org.br/epem/anais/co.html>. Acesso em: 01 maio 2005. 